\title{
Bangladesh Health System and the Millennium Development Goals: Strategic Policy Options for Sustained Progress in Maternal and Child Health
}

\author{
Anwar Islam ${ }^{1, ~ *, ~ T u h i n ~ B i s w a s ~}{ }^{2}$ \\ ${ }^{1}$ School of Health Policy and Management, York University, Toronto, Ontario, Canada \\ ${ }^{2}$ Department of Public Health, North South University, Dhaka, Bangladesh
}

Email address:

anwarhill@yahoo.com (A. Islam), aislam@yorku.ca (A. Islam)

\section{To cite this article:}

Anwar Islam, Tuhin Biswas. Bangladesh Health System and the Millennium Development Goals: Strategic Policy Options for Sustained Progress in Maternal and Child Health. European Journal of Preventive Medicine. Vol. 3, No. 3, 2015, pp. 63-70.

doi: $10.11648 /$ j.ejpm. 20150303.15

\begin{abstract}
The timeline set by the United Nations in achieving the Millennium Development Goals (MDGs) pronounced in the year 2000 is coming to end in 2015. While the United Nations has already came up with a new set of 17 Sustainable Development Goals (SDGs) to follow the MDGs, efforts are underway to assess the success and failures of countries in achieving the MDGs. Bangladesh has made tremendous progress in achieving most of the MDGs including those related to maternal and child health. The maternal mortality ratio and the infant mortality rate in Bangladesh declined from 507 and 149 in 1990 to 209 and 53 in 2010 respectively. However, district level review suggests that numerous constraints and challenges confront the health system in sustaining these achievements. Moreover, desegregated data strongly indicate that the progress is even across different regions of the country. Most importantly, significant gaps still persist between the rich and the poor. For example, while the overall IMR is 43 per 1,000 live births among the highest income quintile, the figure is 84 among the lowest income quintile. In other words, Bangladesh must forcefully address regional disparity and rich-poor gap to ensure sustainable and equitable development in all aspects of health. The study identified a few strategic policy directions for this purpose including aggressive pro-poor and sub-national development planning. To ensure availability of and accessibility to primary health care for all, the study strongly argues for the establishment of community clinics throughout the country equipped with skilled midwives and adequate number of community health workers.
\end{abstract}

Keywords: Millennium Development Goals, Desegregated Data, Community Clinics

\section{Introduction}

Although Bangladesh has made substantial economic progress in recent years, raising its per capita income from about US $\$ 600$ in 2008 to more than US $\$ 958$ by 2014 [1]. The Gross Domestic Product (GDP) of Bangladesh, it may be noted, increased from \$115.3 Billion in 2000 to \$150 Billion by 2010 [2]. Over the last decade, the economy grew at a rate of almost $6 \%$ per annum [2]. However, about $32 \%$ of the population still lives in poverty. According to the 2010 National Income and Expenditure Survey, poverty is more pronounced in rural (35\%) than in urban (21.3\%) areas[2].Rising food prices have exacerbated hardships for many families and pushed an estimated 7.5 million additional Bangladeshis into poverty, for a total of 65 million[3].

Along with its economic growth, Bangladesh has also made significant progress in the health sector registering improvements in all major health indicators - from life expectancy at birth to maternal and child health[4]. It made major inroads in reaching a number of the Millennium Development Goals (MDGs) targets, particularly those pertaining to health. The recent Countdown to 2015report categorizes Bangladesh as among only 16 developing countries in the world on track to achieve significant reductions in the under-five and infant mortality rates (MDG 4). Data from Bangladesh's Sample Vital Registration System (SVRS)suggests that the under-five mortality rate declined from 149 in 1990 to 60 per 1,000 live births in 2007, and according to the Bangladesh Demographic and Health Survey (BDHS 2011) to just 53 per 1,000 live births in 2010[7]. If Bangladesh is able to sustain this pace of progress in reducing under-five mortality rate, it could achieve the under-five 
mortality related MDG 4 targetof 48 per 1,000 live births in2015.

Bangladesh also reduced maternal mortality (MDG 5) from approximately 570 to 209 per 100,000 live births between 1990 and 2011[8], principally by expanding access to family planning services. Infrastructural as well as technological developments of recent decades that saw Bangladesh emerge as a truly "unified" country with intricate interconnected roads, highways and bridges and modern telecommunication facilities (rapid expansion of mobile phones, internet use and electronic media) also contributed significantly to this progress in health. Nevertheless, over the last decade, the pace of progress in women's health has slowed considerably [6, 9-11]. For example, the maternal mortality ratio is still considerable higher in rural (230 per 100,000 live births) than in urban areas (178 per 100,000 live births). Inadequate and uneven progress in improving the status of women, in its many dimensions, as well as the extremely low coverage of skilled delivery services, are the most critical underlying factors in this regard. At its current rate, Bangladesh is likely to achieve an average MMR of 143 per 100,000 live births by 2015, falling a little short of the MDG 5 target. However, MMR would remain considerably higher in rural areas. Moreover, significant inequity persists between the rich and the poor in all major health indicators including MMR and IMR[12]. The challenge for Bangladesh, therefore, is not only to make further improvements in major health indicators but also to ensure equity irrespective of place of residence (rural-urban) and income.

\section{Equity: The Missing Ingredient}

As with the pace of economic growth, the progress made by Bangladesh in health services availability, accessibility and quality also remains highly unequal and uneven. The country's development plans, over the years, vastly improved the lives of certain segments of the population while leaving others increasingly far behind. For example, it is estimated that in 2008 the maternal mortality ratio was almost $40 \%$ higher in rural areas than in urban areas (393 versus 242 per 100,000 live births respectively). Similarly, the infant mortality rate in 2007 was 43 per 1,000 live births among people in the highest income quintile; while the corresponding figure was almost double ( 84 per 1,000 live births) for people in the lowest income quintile (UNICEF Bangladesh, 2010). If concerted efforts are not made to rapidly expand access to quality reproductive and maternal health services, all districts in Bangladesh cannot be expected to reach the MDG 5 target. Consequently, a woman's chance of dying during childbirth would remain substantially unchanged.

Fortunately, Bangladesh is not yet facing the dual epidemics of HIV/AIDS and Tuberculosis. Although Bangladesh has some of the highest numbers of TB cases in the world, it has steadily decreased TB incidence and improved the availability of Directly Observed Treatment, Short-course (DOTS) throughout the country. Consequently, the death rate of TB cases decreased from $58 \%$ in 1990 to $43 \%$ in 2011. The cure rate of TB under DOTS was $73 \%$ in 1994, which has crossed the target by more than $85 \%$ [5]. While Bangladesh is considered an "early achiever" in reducing the incidence and prevalence of tuberculosis, clearly the momentum must be maintained to eliminate pockets of heightened vulnerability, particularly for Multiple Drug Resistant (MDR) TB in urban slums and other poorer segments of the population. Bangladesh is also "on track" to reduce the spread of HIV and AIDS (MDG 6). The HIV prevalence rate is estimated to be $0.1 \%$ in the general population; however the rate for Intravenous Drug Users (IDUs) in the capital city of Dhaka (and a few other cities) has reached $7 \%$ and could soon become a concentrated epidemic [13-16].The overall burden of malaria is quite low in Bangladesh, with $80 \%$ of all cases concentrated in three districts of the Chittagong region[17]. It is evident that in case of communicable diseases like TB, HIV/AIDS and malaria Bangladesh has to address equity issues including regional disparity and pockets of vulnerable populations.

\section{Maternal and Child Health: Priorities for Bangladesh}

To ensure that all districts in Bangladesh achieve the MDG4 target, the coverage and quality of essential health services for women and children must continue to improve, particularly in poor and geographically isolated areas. A particular priority is reducing neonatal mortality rate which currently stands at 32 per 1,000 live births while the target was to reach 21 per 1,000 live births by the year 2015 [6].Another challenge is childhood injuries, especially drowning, which has emerged as a pressing public health issue responsible for a full quarter of the deaths among children between 1-4 years of age [18-20]. A third priority is reducing childhood diarrhea and pneumonia through improved family and community hygiene and care practices and expanded access to appropriate treatment. Although Bangladesh is the birthplace of oral rehydration treatment (ORT), the use of zinc to treat diarrhea has been slow to develop, with only one in ten children in poor households and one in three children in wealthy households receiving both ORT and zinc[21]. Similarly, only one in four children living in the poorest households is brought to a health facility or skilled service provider when showing symptoms of an acute respiratory infection or pneumonia. The majority of children suffering from ARI symptoms are instead taken to a local pharmacy or a traditional doctor. These informally or partly trained practitioners, such as the village doctors and the drug retail outlets, are deeply embedded in the community and are usually the first point of contact for many families seeking care for common illnesses.

Underlying Bangladesh's ongoing maternal and child health challenges is a stubbornly high prevalence of adult and child under-nutrition[22]. Over $51 \%$ of children under-five years of age are underweight (BDHS-2011) and anemia affects around $49.6 \%$ of pregnant women and prevalence of anaemia among the women comparatively high in rural than 
urban(44.7\% Vs 36.1\%)[6]. The poor nutritional status of girls, coupled with high rates of child marriage, sustain Bangladesh's high rates of low birth weight $(22 \%)$, neonatal and maternal mortality [23]. Reducing malnutrition and low birth weight will therefore require a lifecycle approach that encompasses child and adolescent development as well as targeted nutrition interventions for vulnerable populations.

\section{The Study in Three Districts: Assessing the Public Health System}

In 2009, the Government of Bangladesh conducted a systematic assessment of constraints affecting the coverage of priority maternal, newborn and child health interventions[24]. The equity-driven study included in-depth analyses of three "under-performing" districts with highly vulnerable populations, as well as a national analysis. The selected districts were Bandarban in the Chittagong division, Jamalpur in the Dhaka division, and Narail in the Khulna division)[2526]. The methods for the assessment, were based on a model developed by Tanahashi and others and refined by UNICEF and the World Bank, and have been described elsewhere [7]. With financial and technical support from the local UNICEF, the study was conducted by the James P Grant School of Public Health, at BRAC University in Dhaka. The country WHO office also provided technical support.

\section{The Study Findings: Constraints and Opportunities of the Public Health System}

Four cross-cutting constraints hindering the expansion of multiple interventions delivered at community, outreach, and facility levels emerged from this analysis. The first and most critical is a severe lack of appropriately trained and equitably deployed human resources in Bangladesh, resulting from outdated human resource policies. For example, a large proportion of sanctioned posts for nurses, doctors, and paramedics - more than $50 \%$ in the most remote districts - are routinely vacant. In under-performing districts, approximately one in three hospitals serving as comprehensive emergency obstetric care referral sites actually has a full emergency obstetric care team, and many are affected by high rates of staff absenteeism. Another reason for inequitable distribution and performance of health professionals is an almost complete lack of appropriate living quarters for remotely posted workers, as well as insufficient security, water and electricity at many health facilities. This serves as a disincentive for health workers to remain in remote posts and for patients to seek care at public health facilities, and also compromises service quality.

The number of sanctioned posts for health workers in Bangladesh is usually calculated according to the number of facilities in a given district or sub-district (upazilla), rather than the density or needs of the population. A uniform formula for human resource need projections throughout the country may be inappropriate, as certain districts have more challenging geography and different population density than the national average. For example, in the hilly and remote district of Bandarban, in the Chittagong division near the Myanmar border, less than 25 percent of roads are paved and another 25 percent are actually waterways for most or all of the year. This complicates the delivery of outreach services and restricts care-seeking behaviors, particularly as more than $35 \%$ of the population in Bandarban lives in extreme poverty and may not be able or willing to pay for non-emergency transportation. Moreover, the population in Bandarban is widely dispersed: compared to the national average of 1,126 per square kilometer, Bandarban has a population density of only about 65 per square kilometer. Local planners have proposed that Bandarban establish new standards for clinic and health worker distribution to better respond to local needs.

The performance of outreach services, such as childhood immunizations, vitamin A supplementation, and family planning, is generally quite strong in Bangladesh. Nearly all infants $(86 \%)$ receive the BCG vaccine and the contraceptive prevalence rate has jumped from just $30 \%$ in 1990 to nearly $61 \%$ among women aged 25-40 as of 2011 as a result of concerted efforts to increase family planning outreach services[6]. Coverage of family planning varies considerably by division, however. In the socially-conservative district of Sylhet, the contraceptive prevalence rate (CPR) is just under $25 \%$, whereas it is over $50 \%$ in the more liberal Khulna and Rajshahi districts.

Challenges in transporting essential supplies and personnel to remote areas of the country, particularly during the rainy season, is another major impediment to delivering care to the most vulnerable populations, particularly when repeat services are required on a fixed schedule, such as childhood immunizations or antenatal care. For example, BCG coverage is $96 \%$ in Bandarban, while complete immunization coverage is just $74 \%$.

A final challenge is low demand for many health services, which is sustained by financial and geographic barriers, but based upon a continuing preference for traditional healers and home management of medical conditions, particularly for women. Socio-cultural dynamics, such as prohibitions against women receiving medical care from male providers and intercaste prejudices, also affect care-seeking behaviors. For example, $51 \%$ of women seek antenatal care at least once during their pregnancies, but only $21 \%$ return for the recommended four visits and only $16 \%$ deliver in a health facility. Institutional delivery drops to below $5 \%$ in the poorest populations, and to below $3 \%$ among women with no education.

According to Bangladesh health and Demography survey 2011 BCG coverage was $98 \%$, but only $86 \%$ of infants were fully immunized with coverage of complete immunization[6].In 2005, Bangladesh conducted an assessment of its childhood immunization program. At the time fully immunized coverage ranging from $83 \%$ in highperforming districts to just $44 \%$ in the most vulnerable and hard-to-reach districts. The reasons behind this inconsistent 
and inequitable immunization coverage were found to be quite similar to those revealed in the 2010 health system assessment: poor district-level planning and program monitoring, inadequate numbers and supervision of health workers, and weak community mobilization to build and sustain demand for all childhood immunizations. Based on this analysis, Bangladesh identified 15 low-performing districts for focused health systems strengthening efforts. Elements of the resulting "Reach Every District" (RED) strategy include detailed subdistrict micro-planning, monthly data analysis and quarterly district-level review meetings, "crash" programs for the most hard-to-reach areas, improved supervision of health workers, placement of volunteers where human resources were inadequate, and strengthened community mobilization. The results of Bangladesh's RED program are now well known: in just four years, the coverage of complete immunization increased from $52 \%$ to $74 \%$ and geographic disparities have been nearly eliminated. Today, more than 184,000 additional infants have been vaccinated compared with 2005 levels. The RED strategy also had a positive impact on vitamin A supplementation and tetanus toxoid injections for pregnant women, which are delivered through the same outreach programs.

This successful example of how Bangladesh has been able to rapidly expand the coverage of a critical health intervention is evidence of what could be accomplished between now and 2015 through well-prioritized health systems strengthening efforts. It is also one of the most compelling examples of missed opportunities to efficiently scale up the coverage of other key interventions, such as ORS and zinc.

The remaining $26 \%$ of children who are still not consistently reached through routine outreach services, even after implementation of the RED strategy, should help us to identify Bangladesh's most vulnerable households and districts. Reaching this last $26 \%$ of the population with priority health interventions should be Bangladesh's utmost priority to further reduce child and maternal mortality rates and secure more certain and equitable MDG attainment.

\section{The Study Outcome: Priority Health Interventions for Bangladesh}

The results of the Bangladesh health system assessment enabled national health officials to identify interventions and health systems strengthening priorities that were felt to be most appropriate to the local context. Consultations were held at both national and district levels, with the understanding that district-level plans and norms may need to be modified from the national models to fit local circumstances under improved decentralized planning and management. Only interventions with a strong evidence base were considered for scale up.

Public health officials and technical partners identified four related packages of newborn and child health interventions for focused scale-up in Bangladesh. These are 1) community-based sanitation and hygiene promotion, 2) injury prevention programs, 3) treatment of diarrhea and pneumonia at the community level (with ORS, zinc, and antibiotics), and 4) improved community-based care and referral systems for sick newborns. All of these services are currently available at public facilities, but with highly variable levels of coverage, quality, and geographic accessibility. Given the high burden of diarrheal diseases and ARIs, the option of adding pneumococcal and rotavirus vaccines to Bangladesh's existing immunization programs was also strongly recommended. Three additional priorities were identified for women's health, namely: 1) continuing to increase use of modern family planning methods, 2) making safe delivery and basic emergency obstetric and neonatal care available at the union level, and 3) increasing demand for antenatal care and institutional delivery through vouchers or other demand generation programs for the poor.

Such modifications to Bangladesh's primary care system will require improved models for community and outreach services anchored by needs-based, pro-poor human resource policies and strengthened monitoring and supervisory mechanisms. Expanded community mobilization and behavior change programs, often neglected in health systems design, are necessary to increase the uptake of new services. Primary care and normal delivery services must also be priced competitively with traditional medical providers and local drug sellers.

\section{Potential Impacts of Scaling up Priority Interventions}

Drawing upon the results of the health systems assessment, a number of health systems strengthening scenarios were modeled and compared according to marginal cost and impact using the computer-based World Bank / UNICEF developed Marginal Budgeting for Bottlenecks (MBB) tool [8]. Impact projections were calculated using the Lives Saved Tool (LiST).

\section{"Best Buys" for Bangladesh}

Option 1: Community Clinics

Community clinics: Essential primary care, including community case management for childhood diarrhea, pneumonia, and neonatal infections

Staffing: 1 community health worker for 2,000 population; community clinics allocated based on geography as well as population.

Outreach: Childhood immunizations, including rotavirus and pneumococcal vaccines

Special focus on equity: Hardship allowances for health workers in remote areas, pay for performance schemes to reinforce quality standards at community clinics.

Results: 90\% MDG 1, 104\% MDG 4, 81\% MDG 5

The results indicate that aggressively pursuing an expanded version of the Government of Bangladesh's new 
community clinic scheme could lead to equitable MDG 4 attainment in Bangladesh by 2015 . While the existing model envisions one clinic for every 6,000 people, the sub-national health system assessment suggests that the ratio should be adjusted to a district's geography, such that there is one clinic for every 2,000 people in the most hard-to-reach areas of the country and one for every 6,000 elsewhere. To ensure the availability of a more comprehensive continuum of care for the most vulnerable populations, and to capitalize on the new infrastructure, community clinic services should be upgraded to include community-based treatment and referral of neonatal infections, diarrhea, pneumonia and malnutrition, as well as counseling on hygiene and infant and young child feeding. According to district officials, community clinic expansion is more likely to be successful if complemented by focused investments in community-based human resources, including expanded recruitment schemes and the introduction of incentives, such as hardship allowances and pay-forperformance systems, to encourage health professionals to work in remote areas. Investments in human resources should be complemented by investments in community mobilization and behavior change, to build demand for the community clinics instead of (or in addition to) traditional medical providers and local drug sellers.

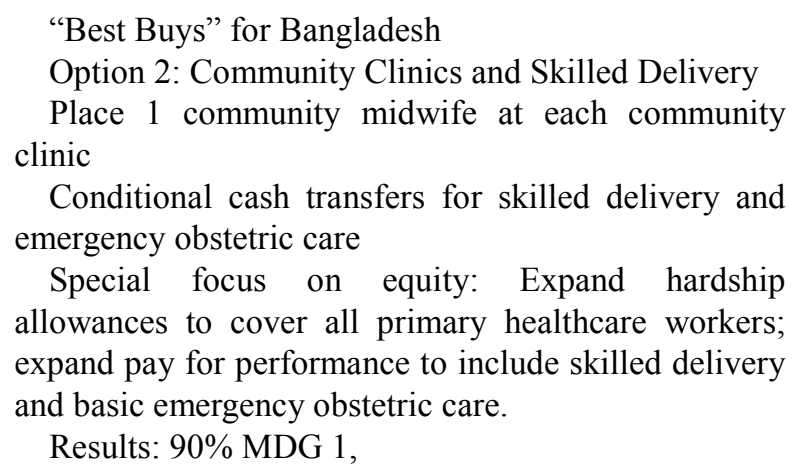

Based upon conservative five-year targets set by the Government of Bangladesh and stakeholders for this scenario, equitably- allocated community clinics could reduce deaths due to diarrhea by $72 \%$, pneumonia by $45 \%$, and neonatal deaths by $44 \%$. This would translate into more than 107,000 under-five deaths averted and a new under-five mortality rate of 29, exceeding the MDG 4 target. This scenario would also enable the most vulnerable districts achieve the MDG 4 targets by 2015, rather than exacerbating existing disparities. For example, the Bandarban district is currently off-track to achieve MDG 4, but it could reduce the under-five mortality rate from 79 to 49 through this strategy. Decreases in diarrhea and childhood illnesses, combined with improved household feeding practices, could also have a positive impact on Bangladesh's high rates of childhood under nutrition, preventing more than 30,000 cases of stunting. Finally, expanded access to family planning and antenatal care, plus cash transfers to support skilled delivery, could reduce maternal mortality by $35 \%$. This is equivalent to approximately $81 \%$ of MDG 5 , compared with just $52 \%$ at the current rate of progress.

To achieve more sizable impacts on maternal health at a national scale will require aggressive investments in facility infrastructure, human resources, and training. Even if such investments and renovations could be realized in the next five years, changing the population's widespread preference for home delivery (more than eight in ten women delivers at home in Bangladesh) to achieve coverage levels sufficient for MDG 5 attainment will probably require much more time. Based on the results of the health system assessment, Bangladesh officials were interested to know how much progress could be achieved by improving the quality and use of existing facilities and posting trained community midwives at community clinics. A second scenario was therefore modeled, which includes 3,000 additional community midwives, modest investments in upazilla health center infrastructure and equipment, and expanded community mobilization programs for reproductive health. As before, this model also includes incentives for health professionals to work in rural areas and cash transfers to encourage women to use skilled delivery services. Based upon estimated new coverage frontiers for skilled delivery and emergency obstetric care, this strategy could reduce maternal mortality by $44 \%$ nationally by 2015 (88\% of MDG5), saving the lives of close to 6,700 women in five years.

\section{Estimated Costs of Priority Interventions Scale-Up}

The two health systems strengthening scenarios presented above were designed to improve the quality of health services that are already publicly available in Bangladesh, or that are under strong consideration for national expansion. They were developed through extensive national and subnational discussions about Bangladesh's health priorities and systemic health systems constraints and draw upon refined evidence about how health systems constraints vary from district to district. Both scenarios were therefore deemed to be realistic options for health programming in Bangladesh over next five years, provided sufficient resources could be made available. The greatest challenge in implementing either strategy is likely to be accelerating the recruitment and training of community health workers and nurses: the current coverage gap is nearly 30,000 community health workers (family welfare assistants and health assistants) and 3,000 nurses, yet Bangladesh's current training programs only produce approximately 200 new community health workers and 400 new nurses per year. While the community health workers are being trained by the private sector (primarily the NGOs), the nurses (non-graduate level) are the products of poorly organized public sector nursing institutes scattered around the country.

Marginal cost estimates suggest that community clinics and related community services could be expanded for an 
additional investment of just $\$ 2.69$ per capita per year between 2011 and 2015. These investments would need to be allocated according to the population's needs rather than distributed equally in the population. For example, Bandarban district would require just over $\$ 7.00$ per capita per year to achieve the strategy's coverage objectives, whereas just $\$ 5.00$ would be required in the Narail district, where the population is poorer but more concentrated and geographically accessible. Adding community midwives to community clinics and improving safe delivery and basic emergency obstetric care services at upazilla health centers would bring the price to approximately $\$ 4.69$ per capita per year, which is a considerable increase but could enable Bangladesh to nearly attain all the health-related MDGs.

These comparisons suggest that the most efficient use of additional health resources in Bangladesh over the next five years may be to combine expanded community-based services with complementary efforts to improve the quality of services already offered at primary care facilities through financial strategies such as hardship allowances and pay-forperformance policies. For faster and more equitable results, health systems strengthening efforts should focus strongly on underperforming districts known to have large vulnerable populations. Changing behavior is perhaps the most critical strategy to reduce the three top killers of young children: diarrhea, pneumonia and neonatal conditions. Health education could also cover the prevention of injuries and drowning, which is another major cause of death not fully captured in this analysis. The combined approaches of targeting the most vulnerable and emphasizing behavior change could ensure that progress in maternal and child health is both sustainable and impacts a broader population, including - in particular-adolescent girls.

Finally, it is worth noting that the marginal cost estimates for community- and primary care-focused strategies are considerably less expensive in Bangladesh than in other countries in South Asia and Sub-Saharan Africa with similar baseline coverage, and could impact a population of over 18 million children under five and 43 million women of reproductive age.

\section{Conclusions}

The District Investment Case Analysis proved to be an extremely useful tool in identifying health system bottlenecks at the district level. The participatory nature of the exercise made the tool sensitive to differential local realities making it possible to identify district-specific health system bottlenecks On the other hand, it was clearly apparent that World Bank and UNICEF developed the Marginal Budgeting for Bottlenecks tool, with some minor modifications, could be effectively applied in Bangladesh in costing out the additional funding requirements in addressing the identified system bottlenecks. In other words, easily adaptable and applicable tools are available to identify system bottlenecks in achieving the health-related MDGs and in determining the resource requirements in overcoming them.
The exercise identified a number of cross-cutting health system bottlenecks. These include the scarcity of appropriately trained adequate number of human resources for health and their mal-distribution across Bangladesh. In remote areas, recruitment and retention of key health personnel especially physicians seem to be one of the most critical constraints faced by the public health facilities at the local level. Ensuring the availability of essential drugs, supplies and commodities at the sub-district level, especially in remote areas, has been identified as another health system constraint. At the same time, low demand for health care services is also a critical problem reflecting weak health education/health promotion activities. Clearly communities lack awareness about and information on health and health care.

The MBB exercise clearly shows that effective interventions could be put in place with little additional cost. It seems that the combined additional cost of introducing these interventions nationally will not be more than $\$ 3.00$ per capita per year between 2011 and 2015. However, the cost will be somewhat higher (varying from $\$ 2.00$ to $\$ 4.00$ per capita) in remote and hard-to-reach areas. Given the expected impact on reducing maternal (by $41 \%$ nationally) mortality, under-five (by more than 34\%) mortality and improving neonatal, maternal and child health, the additional cost is quite minimal. Most importantly, introduction of these interventions will significantly strengthen the equitable access to and availability of essential health care services across the country. The additional funds required for these interventions could be mobilized through efficient reallocation of existing resources as well as additional donor contribution.

\section{Summarizing the Priority Strategies}

It was strongly felt that whatever interventions are introduced the diversity of Bangladesh must be recognized and reflected in planning. Consequently, decentralized planning and management that fully take into account local realities are essential to ensure success of the health interventions. Decentralization must be meaningful and must involve communities in the real sense of the term.

Given the persistently high rates of infant and child mortality, the priority interventions include community-based sanitation and hygiene promotion; expanded access to ORS, zinc and antibiotics; and improved community-based care and referral systems for sick newborn. Although these services are widely available throughout Bangladesh, the challenge is to promote equitable scale-up of community and outreach services anchored in needs-based, pro-poor human resource policies as well as strengthened monitoring and supervisory mechanisms. At the same time, to address the complex issues underlying maternal and newborn health, expanding access to safe delivery and basic emergency obstetric and neonatal care would be a critical priority. This will involve a number of interconnected activities including facility renovations and improved human resource policies. 
Further increasing demand for improved maternal and newborn care through a demand-side financing voucher scheme targeting the poor would be essential in this respect.

\section{Limitations of the Study}

The study was conducted in only three districts in Bangladesh. Although they were from different diverse geographical areas of Bangladesh, they do not necessarily represent the country. The conclusions reached, therefore, should not be generalized and be treated with caution. Moreover, the bottlenecks identified primarily reflect the views expressed by public sector health care service providers. Neither the health service consumers nor private sector service providers, including NGOs, were consulted. These shortcomings of the study should be borne in mind while considering the conclusions reached and/or the priority interventions recommended.

\section{Policy Considerations}

Clearly Bangladesh would require bold policy initiative in further expanding and strengthening its maternal, newborn and child health services in order to achieve the health related MDGs. Policies must be pro-poor and must address the differential local realities. Massive and meaningful decentralization of planning and management of health and health care must be central to such policy change.

\section{Donor Considerations/ Aid Harmonization}

Although the government of Bangladesh could introduce these priority intervention through judicious reallocation of existing resources and investing some additional resources, donor support is critical to successfully address these challenges. Donor support is not only needed for additional funds, but also for technical assistance in implementing the priority interventions. Perhaps donors could make the greatest contribution in the area of policy change/redirection (pro-poor policies and evidenceinformed policies) and bold meaningful decentralization (with effective local level planning and management of the health care system). Donors must also work together, complement each other and coordinate/harmonize their effort.

\section{Epilogue}

The government of Bangladesh has already introduced Community Clinics at the village level to bring health care services closer to the people. The plan is to have 1 community clinic for every 6,000 people. By the end of 2014, a total of 18, 000 Community Clinics have been made operational. Community Clinics are bringing revolutionary change in the public health system by making primary health care services easily accessible to the people at large. Nevertheless, many shortcomings of the Community Clinics remain including shortages of adequately trained human resources, supply of essential drugs and functional interrelationships between the Community Clinics and the Upazila Health Complexes - the hub of the primary health care system. Some of the recommendations of this study could be implemented to further strengthen the Community Clinics. It would take another study to comprehensively review the strengths and weaknesses of the Community Clinics and suggest remedial actions.

\section{Acknowledgment}

The study was conducted under the overall supervision of the principal author while he was serving as a Professor and Director of the James P Grant School of Public Health, BRAC University. The authors acknowledge the contributions made by the team members engaged in the study. Some technical experts from the local UNICEF also made significant contributions in designing, implementing and reviewing the study. The authors are deeply grateful to them for their excellent professional help and contribution.

\section{References}

[1] http://data.worldbank.org/indicator/NY.GDP.PCAP.CD.

[2] http://data.worldbank.org/indicator/NY.GDP.MKTP.CD

[3] Sharma, P., Regional food security from a human security perspective.

[4] Demographic, B., Health Survey BDHS (2011). Preliminary report. NIPORT, 2013.

[5] General Economics Division (GED) Bangladesh Planning Commision, G.o.t.p.s.R.B., June 2013, Millennium Development Goals: Bangladesh pogress report 2012.

[6] Chen, L.C., M. Rahman, and A. Sarder, Epidemiology and causes of death among children in a rural area of Bangladesh. International journal of epidemiology, 1980. 9(1): p. 25-34.

[7] Islam, A. and T. Biswas, Health System Bottlenecks in Achieving Maternal and Child Health-Related Millennium Development Goals: Major Findings from District Level in Bangladesh. Journal of US-China Medical Science, 2014. 11(3). p. 147-158.

[8] Knippenberg, R., A. Soucat, and W. Vanlerberghe, Marginal Budgeting for Bottlenecks: A tool for performance based planning of health and nutrition services for achieving Millennium Development Goals. World Bank, UNICEF, WHO, 2003.

[9] Koenig, M.A., et al., Maternal health and care-seeking behavior in Bangladesh: findings from a national survey. International Family Planning Perspectives, 2007: p. 75-82.

[10] Hardee, K., et al., Reproductive health policies and programs in eight countries: progress since Cairo. International Family Planning Perspectives, 1999: p. S2-S9.

[11] Tinker, A.G., K. Finn, and J. Epp, Improving Women's Health. The World Bank, Washington DC, 1998. 
[12] Khan, M.M.H., et al., Trends in sociodemographic and healthrelated indicators in Bangladesh, 1993-2007: will inequities persist? Bulletin of the World Health Organization, 2011. 89(8): p. 583-593.

[13] Bond, V. and L. Nyblade, The importance of addressing the unfolding TB - HIV stigma in high HIV prevalence settings. Journal of Community \& Applied Social Psychology, 2006. 16(6): p. 452-461.

[14] Dayton, J.M. and M.H. Merson, GLOBAL DIMENSIONS OF THE AIDS EPIDEMIC:: Implications for Prevention and Care. Infectious disease clinics of North America, 2000. 14(4): p. 791-808.

[15] Lönnroth, K., et al., Drivers of tuberculosis epidemics: the role of risk factors and social determinants. Social science \& medicine, 2009. 68(12): p. 2240-2246.

[16] World health Organization. World health statistics 2010.

[17] Russell, S., The economic burden of illness for households in developing countries: a review of studies focusing on malaria, tuberculosis, and human immunodeficiency virus/acquired immunodeficiency syndrome. The American journal of tropical medicine and hygiene, 2004. 71(2 suppl): p. 147-155.

[18] Hyder, A.A., Childhood injuries: defining a global agenda for research and action. African safety promotion, 2006. 4(1): p. p. 87-95.

[19] Rahman, A., et al., Analysis of the childhood fatal drowning situation in Bangladesh: exploring prevention measures for low-income countries. Injury prevention, 2009. 15(2): p. 7579 .
[20] Hyder, A.A., et al., Childhood drowning in low - and middle - income countries: Urgent need for intervention trials. Journal of paediatrics and child health, 2008. 44(4): p. 221227.

[21] Larson, C.P., U.R. Saha, and H. Nazrul, Impact monitoring of the national scale up of zinc treatment for childhood diarrhea in Bangladesh: repeat ecologic surveys. PLoS medicine, 2009. 6(11): p. e1000175.

[22] Arulampalam, W., A. Bhaskar, and N. Srivastava, Does Greater Autonomy among Women Provide the Key to Better Child Nutrition. 2012, Swindon, UK: Economic and Social Research Council.

[23] Chen, L.C., M. Rahman, and A. Sarder, Epidemiology and causes of death among children in a rural area of Bangladesh. International journal of epidemiology, 1980. 9(1): p. 25-34.

[24] Bhutta, Z.A., et al., Countdown to 2015 decade report (200010): taking stock of maternal, newborn, and child survival. The Lancet, 2010. 375(9730): p. 2032-2044.

[25] Islam, A. and T. Biswas, Health System Bottlenecks in Achieving Maternal and Child Health-Related Millennium Development Goals: Major Findings from District Level in Bangladesh. Journal of US-China Medical Science, 2014. 11(3). p. 147-158.

[26] Knippenberg, R., A. Soucat, and W. Vanlerberghe, Marginal Budgeting for Bottlenecks: A tool for performance based planning of health and nutrition services for achieving Millennium Development Goals. World Bank, UNICEF, WHO, 2003. 